\title{
A DICHOTOMY FOR HIGHER-DIMENSIONAL FLOWS
}

\author{
A. ARBIETO AND C. A. MORALES \\ (Communicated by Yingfei Yi)
}

\begin{abstract}
We analyze the dichotomy between sectional-Axiom A flows and flows with points accumulated by periodic orbits of different indices. Indeed, this is proved for $C^{1}$ generic flows whose singularities accumulated by periodic orbits have codimension one. Our result improves the work of M. J. Pacifico and the second author.
\end{abstract}

\section{INTRODUCTION}

Mañé discussed in his breakthrough work [17] if the star property, i.e., the property of being far away from systems with nonhyperbolic periodic orbits, is sufficient to guarantee that a system be Axiom A. Although this is true for diffeomorphisms [12], it is not for flows due to the geometric Lorenz attractor [2], [10, [11. On the other hand, if singularities are not allowed, then the answer turns out to be positive by [6]. Previously, Mañé connects the star property with the nowadays called Newhouse phenomenon, at least for surfaces. In fact, he proved that a $C^{1}$ generic surface diffeomorphism either is Axiom A or displays infinitely many sinks or sources 18 . In the extension of this work on surfaces, 22 obtained the following results about $C^{1}$-generic flows for closed 3-manifolds: Any $C^{1}$-generic star flow is singular-Axiom $\mathrm{A}$ and, consequently, any $C^{1}$-generic flow is singular-Axiom $\mathrm{A}$ or displays infinitely many sinks or sources. The notion of singular-Axiom $A$ was introduced in [23], inspired on the dynamical properties of both Axiom A flows and the geometric Lorenz attractor. It is then natural to investigate such generic phenomena in higher dimensions, and the natural challenges are: Is a $C^{1}$-generic star flow in a closed $n$-manifold singular-Axiom A? Does a $C^{1}$-generic vector field in a closed $n$-manifold have singular-Axiom $\mathrm{A}$ or does it have infinitely many sinks or sources? Unfortunately, what we know is that the second question has a negative answer for $n \geq 5$, as counterexamples can be obtained by suspending the diffeomorphisms in Theorem $\mathrm{C}$ of [3] (but the answer may be positive for $n=4$ ).

A new light comes from the notion of a sectional-hyperbolic set and a sectionalAxiom A flow introduced in [20]. For instance, on p. 947 in 9 ] it is conjectured that the chain recurrent set of every star flow on a closed manifold is sectionalhyperbolic up to flow-reversing (see Definition 1.2 on p. 946 of [9]). However, such a conjecture must be reformulated since the gluing of a Lorenz attractor and a Lorenz repeller (cf. p. 1576 in [22]) provides an open set of star flows in $S^{3}$ for

Received by the editors August 8, 2011 and, in revised form, November 7, 2011.

2010 Mathematics Subject Classification. Primary 37D30; Secondary 37C10.

Key words and phrases. Sectional-Axiom A, Morse index, vector field.

This work was partially supported by CNPq, CAPES-Prodoc, FAPERJ and PRONEX/DS from Brazil. 
which the chain recurrent set is not sectional-hyperbolic up to flow-reversing. A possiblity for such a reformulation suggested by the first author is to replace the term singular-Axiom A by sectional-Axiom A in [22]. In this way we obtain the following conjecture in dimension $n \geq 3$ :

Conjecture 1. $C^{1}$-generic star flows on closed $n$-manifolds are sectional-Axiom A.

Analogously we can ask if a $C^{1}$-generic vector field in a closed $n$-manifold is sectional-Axiom A or displays infinitely many sinks or sources. But now the answer is negative not only for $n=5$, by the suspension of [3] as above, but also for $n=4$ by [26] and the suspension of certain diffeomorphisms [19]. Nevertheless, in all these counterexamples, it is possible to observe the existence of points accumulated by hyperbolic periodic orbits of different Morse indices. Since such a phenomenon can also be observed in a number of well-known examples of nonhyperbolic systems and since, in dimension three, that phenomenon implies the existence of infinitely many sinks or sources, it is possible to formulate the following dichotomy (which, by virtue of Proposition 13, follows from Conjecture 10:

Conjecture 2. $C^{1}$-generic vector fields $X$ satisfy (only) one of the following properties:

(1) X has a point accumulated by hyperbolic periodic orbits of different Morse indices;

(2) $X$ is sectional-Axiom $A$.

In this paper we prove Conjecture 2 but in a case very close to the threedimensional one, namely, when the singularities accumulated by periodic orbits have codimension one (i.e. Morse index 1 or $n-1$ ). Observe that our result implies the dichotomy in [22] since the assumption about the singularities is automatic for $n=3$. It also implies Conjecture 2 in large classes of vector fields as, for instance, those whose singularities (if any) have codimension one. As an application we prove Conjecture 1 for star flows with spectral decomposition as soon as the singularities accumulated by periodic orbits have codimension one. Let us state our results in a precise way.

In what follows $M$ is a compact connected boundaryless Riemannian manifold of dimension $n \geq 3$ (a closed $n$-manifold for short). If $X$ is a $C^{1}$ vector field in $M$, we will denote by $X_{t}$ the flow generated by $X$ in $M$. A subset $\Lambda \subset M$ is invariant if $X_{t}(\Lambda)=\Lambda$ for all $t \in \mathbb{R}$. By a closed orbit we mean a periodic orbit or a singularity. We define the omega-limit set of $p \in M$ by

$$
\omega(p)=\left\{x \in M: x=\lim _{n \rightarrow \infty} X_{t_{n}}(p) \text { for some sequence } t_{n} \rightarrow \infty\right\}
$$

and call $\Lambda$ transitive if $\Lambda=\omega(p)$ for some $p \in \Lambda$. Clearly, every transitive set is compact invariant. As is customary, we call $\Lambda$ nontrivial it it does not reduce to a single orbit.

Denote by $\|\cdot\|$ and $m(\cdot)$ the norm and the minimal norm induced by the Riemannian metric and by $\operatorname{Det}(\cdot)$ the jacobian operation. A compact invariant set $\Lambda$ is hyperbolic if there are a continuous invariant tangent bundle decomposition

$$
T_{\Lambda} M=\hat{E}_{\Lambda}^{s} \oplus E_{\Lambda}^{X} \oplus \hat{E}_{\Lambda}^{u}
$$


and positive constants $K, \lambda$ such that $E_{\Lambda}^{X}$ is the subbundle generated by $X$,

$$
\left\|D X_{t}(x) / \hat{E}_{x}^{s}\right\| \leq K e^{-\lambda t} \quad \text { and } \quad m\left(D X_{t}(x) / \hat{E}_{x}^{u}\right) \geq K^{-1} e^{\lambda t},
$$

for all $x \in \Lambda$ and $t \geq 0$. Sometimes we write $\hat{E}_{x}^{s, X}, \hat{E}_{x}^{u, X}$ to indicate dependence on $X$.

A closed orbit $O$ is hyperbolic if it is as a compact invariant set. In such a case we define its Morse index $I(O)=\operatorname{dim}\left(\hat{E}_{O}^{s}\right)$, where $\operatorname{dim}(\cdot)$ stands for the dimension operation. If $O$ reduces to a singularity $\sigma$, then we write $I(\sigma)$ instead of $I(\{\sigma\})$ and say that $\sigma$ has codimension one if $I(\sigma)=1$ or $I(\sigma)=n-1$. It is customary to call a hyperbolic closed orbit of maximal (resp. minimal) a Morse index sink (resp. source).

On the other hand, an invariant splitting $T_{\Lambda} M=E_{\Lambda} \oplus F_{\Lambda}$ over $\Lambda$ is dominated (we also say that $E_{\Lambda}$ dominates $F_{\Lambda}$ ) if there are positive constants $K, \lambda$ such that

$$
\frac{\left\|D X_{t}(x) / E_{x}\right\|}{m\left(D X_{t}(x) / F_{x}\right)} \leq K e^{-\lambda t}, \quad \forall x \in \Lambda \text { and } t \geq 0 .
$$

In this work we agree to call a compact invariant set $\Lambda$ partially hyperbolic if there is a dominated splitting $T_{\Lambda} M=E_{\Lambda}^{s} \oplus E_{\Lambda}^{c}$ with contracting dominating subbundle $E_{\Lambda}^{s}$, namely,

$$
\left\|D X_{t}(x) / E_{x}^{s}\right\| \leq K e^{-\lambda t}, \quad \forall x \in \Lambda \text { and } t \geq 0 .
$$

We stress however that this is not a standard usage (specially due to the lack of symmetry in this definition). Anyway, in such a case, we say that $\Lambda$ has contracting $\operatorname{dimension} d$ if $\operatorname{dim}\left(E_{x}^{s}\right)=d$ for all $x \in \Lambda$. Moreover, we say that the central subbundle $E_{\Lambda}^{c}$ is sectionally expanding if

$$
\operatorname{dim}\left(E_{x}^{c}\right) \geq 2 \quad \text { and } \quad\left|\operatorname{Det}\left(D X_{t}(x) / L_{x}\right)\right| \geq K^{-1} e^{\lambda t}, \quad \forall x \in \Lambda, t \geq 0,
$$

and all two-dimensional subspaces $L_{x}$ of $E_{x}^{c}$.

The notion of a sectional-expanding subbundle naturally suggests the notion of a sectional-hyperbolic set: a partially hyperbolic set whose singularities (if any) are hyperbolic and whose central subbundle is sectionally expanding 1

Now we recall the concept of sectional-Axiom A flow [20]. Call a point $p \in M$ nonwandering if for every neighborhood $U$ of $p$ and every $T>0$ there is $t>T$ such that $X_{t}(U) \cap U \neq \emptyset$. We denote by $\Omega(X)$ the set of nonwandering points of $X$ (which is clearly a compact invariant set). We say that $X$ is an Axiom $A$ flow if $\Omega(X)$ is both hyperbolic and the closure of the closed orbits. In light of this definition it would be natural to study the class of vector fields for which the nonwandering set is both sectional-hyperbolic and the closure of the closed orbits. Unfortunately, such a class is far from being generic among the star flows due to the aforementioned example in [22. Alternatively we observe that, by the Spectral Decomposition Theorem [14], the nonwandering set of any Axiom A flow splits into finitely many disjoint transitive hyperbolic sets with dense closed orbits (i.e. with a dense subset of closed orbits). This observation motivates the following definition:

Definition 3. We say that a $C^{1}$ vector field $X$ is a sectional-Axiom $A$ flow if there is a finite disjoint decomposition $\Omega(X)=\Omega_{1} \cup \cdots \cup \Omega_{k}$ formed by transitive sets with dense closed orbits $\Omega_{1}, \cdots, \Omega_{k}$ such that, for all $1 \leq i \leq k, \Omega_{i}$ is either a hyperbolic set for $X$ or a sectional-hyperbolic set for $X$ or a sectional-hyperbolic set for $-X$.

\footnotetext{
${ }^{1}$ Some authors use the term singular-hyperbolic instead.
} 
Let $\mathcal{X}^{1}$ denote the space of $C^{1}$ vector fields $X$ in $M$. Notice that it is a Baire space if it is equipped with the standard $C^{1}$ topology. The expression $C^{1}$-generic vector field will mean a vector field in a certain residual subset of $\mathcal{X}^{1}$. We say that a point is accumulated by periodic orbits if it lies in the closure of the union of the periodic orbits, and accumulated by hyperbolic periodic orbits of different Morse index if it lies simultaneously in the closure of the hyperbolic periodic orbits of Morse index $i$ and $j$ with $i \neq j$. With these definitions we can state our main result settling a special case of Conjecture 2 .

Theorem A. $A C^{1}$-generic vector field $X$ for which the singularities accumulated by periodic orbits have codimension one satisfies (only) one of the following properties:

(1) X has a point accumulated by hyperbolic periodic orbits of different Morse indices;

(2) $X$ is sectional-Axiom $A$.

Standard $C^{1}$-generic results [4] imply that the sectional-Axiom A flows in the second alternative above also satisfy the no-cycle condition.

The proof of our result follows that of Theorem A in [22]. However, we need a more direct approach that bypasses Conjecture 1. Indeed, we shall use some methods in [22] together with a combination of results [8, 9], 20] for nontrivial transitive sets (originally proved for robustly transitive sets).

Definition $4([1])$. We say that $X$ has spectral decomposition if there is a finite partition $\Omega(X)=\Lambda_{1} \cup \cdots \cup \Lambda_{l}$ formed by transitive sets $\Lambda_{1}, \cdots, \Lambda_{l}$.

Theorem A will imply the following approach to Conjecture 1

Theorem B. A $C^{1}$-generic star flow with spectral decomposition and for which the singularities accumulated by periodic orbits have codimension one is sectionalAxiom A.

\section{Proofs}

Hereafter we fix a closed $n$-manifold $M, n \geq 3, X \in \mathcal{X}^{1}$ and a compact invariant set $\Lambda$ of $X$. Denote by $\operatorname{Sing}(X, \Lambda)$ the set of singularities of $X$ in $\Lambda$. We shall use the following concept from [8].

Definition 5. We say that $\Lambda$ has a definite index $0 \leq \operatorname{Ind}(\Lambda) \leq n-1$ if there are a neighborhood $\mathcal{U}$ of $X$ in $\mathcal{X}^{1}$ and a neighborhood $U$ of $\Lambda$ in $M$ such that $I(O)=\operatorname{Ind}(\Lambda)$ for every hyperbolic periodic orbit $O \subset U$ of every vector field $Y \in \mathcal{U}$. In such a case we say that $\Lambda$ is strongly homogeneous (of index $\operatorname{Ind}(\Lambda)$ ).

It turns out that the strongly homogeneous property imposes certain constraints on the Morse indices of the singularities [9]. To explain this we use the concept of saddle value of a hyperbolic singularity $\sigma$ of $X$ defined by

$$
\Delta(\sigma)=\operatorname{Re}(\lambda)+\operatorname{Re}(\gamma),
$$

where $\lambda$ (resp. $\gamma$ ) is the stable (resp. unstable) eigenvalue with maximal (resp. minimal) real part (cf. [27], p. 725). Indeed, based on Hayashi's connecting lemma [13] and well-known results about unfolding of homoclinic loops [27, Lemma 4.3 in 9] proves that if $\Lambda$ is a robustly transitive set which is strongly homogeneous 
with hyperbolic singularities, then $\Delta(\sigma) \neq 0$ and, furthermore, $I(\sigma)=\operatorname{Ind}(\Lambda)$ or $\operatorname{Ind}(\Lambda)+1$ depending on whether $\Delta(\sigma)<0$ or $\Delta(\sigma)>0, \forall \sigma \in \operatorname{Sing}(X, \Lambda)$. However, we can observe that the same is true for nontrivial transitive sets (instead of robustly transitive sets), for the proof in [9] uses the connecting lemma only once. In this way we obtain the following lemma.

Lemma 6. Let $\Lambda$ be a nontrivial transitive set which is strongly homogeneous with singularities (all hyperbolic) of $X$. Then, every $\sigma \in \operatorname{Sing}(X, \Lambda)$ satisfies $\Delta(\sigma) \neq 0$ and one of the properties below:

- If $\Delta(\sigma)<0$, then $I(\sigma)=\operatorname{Ind}(\Lambda)$.

- If $\Delta(\sigma)>0$, then $I(\sigma)=\operatorname{Ind}(\Lambda)+1$.

On the other hand, the following inequalities for strongly homogeneous sets $\Lambda$ where introduced in 8]:

$$
\begin{array}{ll}
I(\sigma)>\operatorname{Ind}(\Lambda), & \forall \sigma \in \operatorname{Sing}(X, \Lambda), \\
I(\sigma) \leq \operatorname{Ind}(\Lambda), & \forall \sigma \in \operatorname{Sing}(X, \Lambda) .
\end{array}
$$

We shall use the above lemma to present a special case where one of these inequalities can be proved.

Proposition 7. Let $\Lambda$ be a nontrivial transitive set which is strongly homogeneous with singularities (all hyperbolic of codimension one) of $X$. If $n \geq 4$ and $1 \leq$ $\operatorname{Ind}(\Lambda) \leq n-2$, then $\Lambda$ satisfies either (1) or (2).

Proof. Otherwise there are $\sigma_{0}, \sigma_{1} \in \operatorname{Sing}(X, \Lambda)$ satisfying $I\left(\sigma_{0}\right) \leq \operatorname{Ind}(\Lambda)<I\left(\sigma_{1}\right)$. Since both $\sigma_{0}$ and $\sigma_{1}$ have codimension one and $1 \leq \operatorname{Ind}(\Lambda) \leq n-2$, we obtain $I\left(\sigma_{0}\right)=1$ and $I\left(\sigma_{1}\right)=n-1$. If $\Delta\left(\sigma_{0}\right) \geq 0$, then $I\left(\sigma_{0}\right)=\operatorname{Ind}(\Lambda)+1$ by $\operatorname{Lemm} 6$ so $\operatorname{Ind}(\Lambda)=0$, which contradicts $1 \leq \operatorname{Ind}(\Lambda)$. Then $\Delta\left(\sigma_{0}\right)<0$, and so $\operatorname{Ind}(\Lambda)=$ $I\left(\sigma_{0}\right)=1$ by Lemma 6. On the other hand, if $\Delta\left(\sigma_{1}\right)<0$, then $\operatorname{Ind}(\Lambda)=I\left(\sigma_{1}\right)=$ $n-1$ by Lemma 6. As $\operatorname{Ind}(\Lambda)=1$ we get $n=2$, contradicting $n \geq 4$. Then $\Delta\left(\sigma_{1}\right) \geq 0$, so $I\left(\sigma_{1}\right)=\operatorname{Ind}(\Lambda)+1$ by Lemma 6. thus $n=3$, contradicting $n \geq 4$. The proof follows.

The importance of (11) and (2) relies on the the following result proved in [8], [9], 20]: $\mathrm{A} C^{1}$ robustly transitive set $\Lambda$ with singularities (all hyperbolic) which is strongly homogeneous satisfying (11) (resp. (2)) is sectional hyperbolic for $X$ (resp. $-X)$. However, we observed that the same is true for nontrivial transitive sets (instead of robustly transitive sets) as soon as $1 \leq \operatorname{Ind}(\Lambda) \leq n-2$. The proof is similar to that in [8], 9], 20] but uses the so-called preperiodic set 28] instead of the natural continuation of a robustly transitive sets. Combining this with Proposition 7 we obtain the following corollary in which the expression up to flow-reversing (also used in the introduction) means either for $X$ or $-X$.

Corollary 8. Let $\Lambda$ be a nontrivial transitive set which is strongly homogeneous with singularities (all hyperbolic of codimension one) of $X$. If $n \geq 4$ and $1 \leq$ $\operatorname{Ind}(\Lambda) \leq n-2$, then $\Lambda$ is sectional-hyperbolic up to flow-reversing.

A direct application of this corollary is as follows. We say that $\Lambda$ is Lyapunov stable for $X$ if for every neighborhood $U$ of it there is a neighborhood $W \subset U$ of it such that $X_{t}(p) \in U$ for every $t \geq 0$ and $p \in W$. 
It was proved in Theorem $\mathrm{C}$ of 22 that, for $C^{1}$-generic three-dimensional star flows, every nontrivial Lyapunov stable set with singularities is singular-hyperbolic. We will need a similar result for higher-dimensional flows, but with the term singular-hyperbolic replaced by sectional-hyperbolic. The following will supply such a result.

Corollary 9. Let $\Lambda$ be a nontrivial transitive set which is strongly homogeneous with singularities (all hyperbolic of codimension one) of $X$. If $n \geq 4,1 \leq \operatorname{Ind}(\Lambda) \leq$ $n-2$ and $\Lambda$ is Lyapunov stable, then $\Lambda$ is sectional-hyperbolic for $X$.

Proof. By Corollary 8 it suffices to prove that $\Lambda$ cannot be sectional-hyperbolic for $-X$. Assume by contradiction that it does. Then, by integrating the corresponding contracting subbundle, we obtain a strong stable manifold $W_{-X}^{s s}(x), \forall x \in \Lambda$. But $\Lambda$ is Lyapunov stable for $X$, so $W_{-X}^{s s}(x) \subset \Lambda, \forall x \in \Lambda$, contradicting p. 556 in [21]. Then, $\Lambda$ cannot be sectional-hyperbolic for $-X$, and we are done.

We also use Lemma 6 to prove the following proposition.

Proposition 10. Every nontrivial transitive sectional-hyperbolic set $\Lambda$ of a vector field $X$ in a closed $n$-manifold, $n \geq 3$, is strongly homogeneous and satisfies $I(\sigma)=$ $\operatorname{Ind}(\Lambda)+1, \forall \sigma \in \operatorname{Sing}(X, \Lambda)$.

Proof. Since transitiveness implies connectedness we have that the strong stable subbundle $E_{\Lambda}^{s}$ of $\Lambda$ has constant dimension. From this and the persistence of the sectional-hyperbolic splitting we obtain that $\Lambda$ is strongly homogeneous of index $\operatorname{Ind}(\Lambda)=\operatorname{dim}\left(E_{x}^{s}\right)$, for $x \in \Lambda$. Now fix a singularity $\sigma$. To prove $I(\sigma)=\operatorname{Ind}(\Lambda)+1$ we only need to prove that $\Delta(\sigma)>0$ (cf. Lemma 6 ).

Suppose by contradiction that $\Delta(\sigma) \leq 0$. Then, $\Delta(\sigma)<0$ and $I(\sigma)=\operatorname{Ind}(\Lambda)$ by Lemma 6. Therefore, $\operatorname{dim}\left(E_{\sigma}^{s}\right)=\operatorname{dim}\left(\hat{E}_{\sigma}^{s}\right)$, where $T_{\sigma} M=\hat{E}_{\sigma}^{s} \oplus \hat{E}_{\sigma}^{u}$ is the hyperbolic splitting of $\sigma$ (as a hyperbolic singularity of $X$ ). Now, let $W^{s}(\sigma)$ be the stable manifold of $\sigma$ and $W^{s s}(\sigma)$ be the strong stable manifold of $\sigma$ obtained by integrating the strong stable subbundle $E_{\Lambda}^{s}$ (cf. [15]). Notice that $W^{s s}(\sigma) \subset W^{s}(\sigma)$. As $\operatorname{dim}\left(W^{s s}(\sigma)\right)=\operatorname{dim}\left(E_{\sigma}^{s}\right)=\operatorname{dim}\left(\hat{E}_{\sigma}^{s}\right)=\operatorname{dim}\left(W^{s}(\sigma)\right)$, we get $W^{s s}(\sigma)=W^{s}(\sigma)$. But $\Lambda$ is nontrivial transitive, so the dense orbit will accumulate at some point in $W^{s}(\sigma) \backslash\{\sigma\}$. As $W^{s s}(\sigma)=W^{s}(\sigma)$ such a point must belong to $\left(\Lambda \cap W^{s s}(\sigma)\right) \backslash\{\sigma\}$. On the other hand, it is well known that $\Lambda \cap W^{s s}(\sigma)=\{\sigma\}$ (cf. [22]), so we obtain a contradiction which proves the result.

We say that $\Lambda$ is an attracting set if there is a neighborhood $U$ of it such that

$$
\Lambda=\bigcap_{t>0} X_{t}(U)
$$

On the other hand, a sectional-hyperbolic attractor is a transitive attracting set which is also a sectional-hyperbolic set. An unstable branch of a hyperbolic singularity $\sigma$ of a vector field is an orbit in $W^{u}(\sigma) \backslash\{\sigma\}$. We say that $\Lambda$ has dense singular unstable branches if every unstable branch of every hyperbolic singularity on it is dense in $\Lambda$.

The following is a straightforward extension of Theorem D in [22] to higher dimensions (with a similar proof). 
Proposition 11. Let $\Lambda$ be a Lyapunov stable sectional-hyperbolic set of a vector field $X$ in a closed $n$-manifold, $n \geq 3$. If $\Lambda$ has both singularities, all of Morse index $n-1$, and dense singular unstable branches, then $\Lambda$ is a sectional-hyperbolic attractor of $X$.

Now we recall the star flow's terminology from [28].

Definition 12. A star flow is a $C^{1}$ vector field which cannot be $C^{1}$-approximated by ones exhibiting nonhyperbolic closed orbits.

Corollary 9 together with Propositions 10 and 11 implies the key result below.

Proposition 13. A $C^{1}$-generic vector field $X$ on a closed $n$-manifold, $\forall n \geq 3$, without points accumulated by hyperbolic periodic orbits of different Morse indices is a star flow. If, additionally, $n \geq 4$ and every singularity accumulated by periodic orbits has codimension one, then every codimension one singularity of $X$ accumulated by periodic orbits belongs to a sectional-hyperbolic attractor up to flow-reversing.

Proof. We will use the following notation. Given $Z \in \mathcal{X}^{1}$ and $0 \leq i \leq n-1$, we denote by $\operatorname{Per}_{i}(Z)$ the union of the hyperbolic periodic orbits of Morse index $i$. The closure operation will be denoted by $C l(\cdot)$.

Since $X$ has no point accumulated by hyperbolic periodic orbits of different Morse indices, one has

$$
C l\left(\operatorname{Per}_{i}(X)\right) \cap C l\left(\operatorname{Per}_{j}(X)\right)=\emptyset, \quad \forall i, j \in\{0, \cdots, n-1\}, \quad i \neq j .
$$

Then, since $X$ is $C^{1}$-generic, standard lower-semicontinuous arguments (cf. 4]) imply that there are a neighborhood $\mathcal{U}$ of $X$ in $\mathcal{X}^{1}$ and a pairwise disjoint collection of neighborhoods $\left\{U_{i}: 0 \leq i \leq n-1\right\}$ such that $C l\left(\operatorname{Per}_{i}(Y)\right) \subset U_{i}$ for all $0 \leq i \leq$ $n-1$ and $Y \in \mathcal{U}$.

Let us prove that $X$ is a star flow. When necessary we use the notation $I_{X}(O)$ to indicate dependence on $X$. By contradiction assume that $X$ is not a star flow. Then, there is a vector field $Y \in \mathcal{U}$ exhibiting a nonhyperbolic closed orbit $O$. Since $X$ is generic we can assume by the Kupka-Smale Theorem [14] that $O$ is a periodic orbit. Unfolding the eigenvalues of $O$ in a suitable way, we would obtain two vector fields $Z_{1}, Z_{2} \in \mathcal{U}$ of which $O$ is a hyperbolic periodic orbit with $I_{Z_{1}}(O) \neq I_{Z_{2}}(O)$, $1 \leq I_{Z_{1}}(O) \leq n-1$ and $1 \leq I_{Z_{2}}(O) \leq n-1$. Consequently, $O \subset U_{i} \cap U_{j}$, where $i=$ $I_{Z_{1}}(O)$ and $j=I_{Z_{2}}(O)$, which contradicts that the collection $\left\{U_{i}: 0 \leq i \leq n-1\right\}$ is pairwise disjoint. Therefore, $X$ is a star flow.

Next we prove that $C l\left(\operatorname{Per}_{i}(X)\right)$ is a strongly homogeneous set of index $i, \forall 0 \leq$ $i \leq n-1$. Take $Y \in \mathcal{U}$ and a hyperbolic periodic orbit $O \subset U_{i}$ of Morse index $I_{Y}(O)=j$. Then, $O \subset C l\left(\operatorname{Per}_{j}(Y)\right)$, and so $O \subset U_{j}$, from which we get $O \subset$ $U_{i} \cap U_{j}$. As the collection $\left\{U_{i}: 0 \leq i \leq n-1\right\}$ is disjoint, we conclude that $i=j$, and so every hyperbolic periodic orbit $O \subset U_{i}$ of every vector field $Y \in \mathcal{U}$ has Morse index $I_{Y}(O)=i$. Therefore, $C l\left(\operatorname{Per}_{i}(X)\right)$ is a strongly homogeneous set of index $i$.

Now, we prove that every codimension one singularity $\sigma$ accumulated by periodic orbits belongs to a sectional-hyperbolic attractor up to flow-reversing. More precisely, we prove that if $I(\sigma)=n-1$ (resp. $I(\sigma)=1)$, then $\sigma$ belongs to a sectional-hyperbolic attractor of $X$ (resp. of $-X$ ). We only consider that the case $I(\sigma)=n-1$ for the case $I(\sigma)=1$ can be handled analogously by just replacing $X$ by $-X$. 
Since $I(\sigma)=n-1$, one has $\operatorname{dim}\left(W^{u}(\sigma)\right)=1$ and, since $X$ is generic, we can assume that both $C l\left(W^{u}(\sigma)\right.$ ) and $\omega(q)$ (for $q \in W^{u}(\sigma) \backslash\{\sigma\}$ ) are Lyapunov stable sets of $X$ (cf. [5]). As $\sigma$ is accumulated by periodic orbits, we obtain from Lemma 4.2 in 22. that $C l\left(W^{u}(\sigma)\right)$ is a transitive set.

We claim that $C l\left(W^{u}(\sigma)\right)$ is strongly homogeneous. Indeed, since $X$ is generic, the General Density Theorem [25] implies $\Omega(X)=C l(\operatorname{Per}(X) \cup \operatorname{Sing}(X))$. Denote by $\operatorname{Sing}^{*}(X)$ the set of singularities accumulated by periodic orbits. Then, there is a decomposition

$$
\Omega(X)=\left(\bigcup_{0 \leq i \leq n-1} C l\left(\operatorname{Per}_{i}(X)\right)\right) \cup\left(\bigcup_{\sigma^{\prime} \in \operatorname{Sing}(X) \backslash \operatorname{Sing}^{*}(X)}\left\{\sigma^{\prime}\right\}\right)
$$

which is disjoint by (3). In addition, $C l\left(W^{u}(\sigma)\right)$ is transitive, and so it is connected and contained in $\Omega(X)$. As $\sigma \in \operatorname{Sing}^{*}(X)$, by hypothesis we conclude that $C l\left(W^{u}(\sigma)\right) \subset C l\left(\operatorname{Per}_{i_{0}}(X)\right)$ for some $0 \leq i_{0} \leq n-1$. Since we have proved above that $C l\left(\operatorname{Per}_{i_{0}}(X)\right)$ is a strongly homogeneous set of index $i_{0}, C l\left(W^{u}(\sigma)\right)$ is also a strongly homogeneous set of index $i_{0}$. The claim follows.

On the other hand, $X$ is a star flow, and so it has finitely many sinks and sources [16], 24]. From this we obtain $1 \leq i_{0} \leq n-2$, and so $1 \leq \operatorname{Ind}\left(C l\left(W^{u}(\sigma)\right)\right) \leq$ $n-2$. Summarizing, we have proved that $C l\left(W^{u}(\sigma)\right)$ is a transitive set with singularities, all of them of codimension one, which is a Lyapunov stable strongly homogeneous set of index $1 \leq \operatorname{Ind}\left(C l\left(W^{u}(\sigma)\right)\right) \leq n-2$. As certainly $C l\left(W^{u}(\sigma)\right)$ is nontrivial, Corollary 9 applied to $\Lambda=C l\left(W^{u}(\sigma)\right)$ implies that $C l\left(W^{u}(\sigma)\right)$ is sectional-hyperbolic.

Once we have proved that $C l\left(W^{u}(\sigma)\right)$ is sectional-hyperbolic we apply Proposition 10 to $\Lambda=C l\left(W^{u}(\sigma)\right)$, yielding $I\left(\sigma^{\prime}\right)=i_{0}+1, \forall \sigma^{\prime} \in \operatorname{Sing}\left(X, C l\left(W^{u}(\sigma)\right)\right)$. But $\sigma \in C l\left(W^{u}(\sigma)\right)$ and $I(\sigma)=n-1$, so $i_{0}=n-2$ by taking $\sigma^{\prime}=\sigma$ above. Consequently, $I\left(\sigma^{\prime}\right)=n-1$, and so $\operatorname{dim}\left(W^{u}\left(\sigma^{\prime}\right)\right)=1, \forall \sigma^{\prime} \in C l\left(W^{u}(\sigma)\right)$. This implies two things: firstly, that every singularity in $C l\left(W^{u}(\sigma)\right)$ has Morse index $n-1$ and, secondly, since $X$ is generic, we can assume that $C l\left(W^{u}(\sigma)\right)$ has dense unstable branches (cf. Lemma 4.1 in 22$]$ ). So, $C l\left(W^{u}(\sigma)\right)$ is a sectional-hyperbolic attractor by Proposition 11 applied to $\Lambda=C l\left(W^{u}(\sigma)\right)$. Since $\sigma \in C l\left(W^{u}(\sigma)\right)$ we obtain the result.

The last ingredient is the proposition below, whose proof follows from Theorem B of [6], as in the proof of Theorem B, p. 1582, of [22].

Proposition 14. If $n \geq 3$, every $C^{1}$-generic star flow whose singularities accumulated by periodic orbits belong to a sectional-hyperbolic attractor up to flow-reversing is sectional-Axiom A.

Proof of Theorem A. Let $X$ be a $C^{1}$-generic vector field on a closed $n$-manifold, $n \geq 3$, all of whose singularities accumulated by periodic orbits have codimension one. Suppose in addition that there is no point accumulated by hyperbolic periodic orbits of different Morse indices. Since $X$ is $C^{1}$-generic, we have by Proposition 13 that $X$ is a star flow.

If $n=3$, then, since $X$ is generic, Theorem B in 22 implies that $X$ is sectionalAxiom A. 
If $n \geq 4$, then, by Proposition [13, since the singularities accumulated by periodic orbits have codimension one, we have that all such singularities belong to a sectional-hyperbolic attractor up to flow-reversing. Then, $X$ is sectional-Axiom A by Proposition 14

Now we move to the proof of Theorem B.

Hereafter we denote by $W_{X}^{s}(\cdot)$ and $W_{X}^{u}(\cdot)$ the stable and unstable manifold operations [15] with emphasis on $X$. The notation $O(p)$ (or $O_{X}(p)$ to emphasize $X$ ) will indicate the orbit of $p$ with respect to $X$. By a periodic point we mean a point belonging to a periodic orbit of $X$. As usual the notation $\pitchfork$ will indicate the transversal intersection operation.

The following lemma is the flow version of Lemma 15, p. 459, in [7.

Lemma 15. There exists a residual subset $\mathcal{R} \subset \mathcal{X}^{1}$ with the following property: If $X \in \mathcal{R}$ has two periodic points $q_{0}$ and $p_{0}$ such that for any neighborhood $\mathcal{U}$ of $X$ there exists $Y \in \mathcal{U}$ such that the continuations of $q(Y)$ and $p(Y)$ of $q_{0}$ and $p_{0}$ respectively are defined and satisfy $W_{Y}^{s}(O(q(Y))) \pitchfork W_{Y}^{u}(O(p(Y))) \neq \emptyset$, then $X$ satisfies

$$
W_{X}^{s}\left(O\left(q_{0}\right)\right) \pitchfork W_{X}^{u}\left(O\left(p_{0}\right)\right) \neq \emptyset .
$$

We use this lemma to prove the following one.

Lemma 16. A $C^{1}$-generic star flow with spectral decomposition has no points accumulated by hyperbolic periodic orbits of different Morse indices.

Proof. Let $\mathcal{R}$ be the residual subset in Lemma 15. Suppose that $X \in \mathcal{R}$ has spectral decomposition but has no points accumulated by hyperbolic periodic orbits of different Morse indices. Then, there exists $i \neq j$ such that $C l\left(\operatorname{Per}_{i}(X)\right) \cap$ $C l\left(\operatorname{Per}_{j}(X)\right) \neq \emptyset$. Without loss of generality we can assume $i<j$. Take $x \in$ $C l\left(\operatorname{Per}_{i}(X)\right) \cap C l\left(\operatorname{Per}_{j}(X)\right)$ so there are periodic orbits $O\left(p_{0}\right)$ (of index $i$ ) and $O\left(q_{0}\right)$ (of index $j$ ) arbitrarily close to $x$. Clearly $x \in \Omega(X)$, and so there is a basic set $\Lambda$ in the spectral decomposition of $X$ such that $x \in \Lambda$. As the basic sets in the spectral decomposition are disjoint and the orbits $O\left(p_{0}\right), O\left(q_{0}\right)$ are close to $x$ (and belong to $\Omega(X))$, we conclude that $O\left(p_{0}\right) \cup O\left(q_{0}\right) \subset \Lambda$.

Since $\Lambda$ is transitive, the connecting lemma 13 implies that there exists $Y$ arbitrarily close to $X$ such that $W_{Y}^{s}(O(q(Y))) \cap W_{Y}^{u}(O(p(Y))) \neq \emptyset$. On the other hand, $j-i>0$ since $j>i$. Moreover, $\operatorname{dim}\left(W_{Y}^{s}(O(q(Y)))\right)=j+1$ and $\operatorname{dim}\left(W_{Y}^{u}(O(p(Y)))\right)=n-i$ since $i n d(O(q(y)))=j$ and $i n d(O(p(Y)))=i$ (resp.). Then, $\operatorname{dim}\left(W_{Y}^{s}(O(q(Y)))\right)+\operatorname{dim}\left(W_{Y}^{u}(O(p(Y)))\right)=j+1+n-i>n$, and so with another perturbation we can assume that the above intersection is transversal. Since $X \in \mathcal{R}$ we conclude by Lemma 15 that

$$
W_{X}^{s}\left(O\left(q_{0}\right)\right) \pitchfork W_{X}^{u}\left(O\left(p_{0}\right)\right) \neq \emptyset .
$$

Now, using the connecting lemma again, there exists $Y$ close to $X$ with a heterodimensional cycle. But this contradicts the nonexistence of heteroclinic cycles for star flows (cf. Theorem 4.1 in [6]). The proof follows.

Proof of Theorem B. Apply Theorem A and Lemma 16. 


\section{REFERENCES}

[1] Abdenur, F., Generic robustness of spectral decompositions, Ann. Sci. École Norm. Sup. (4) 36 (2003), no. 2, 213-224. MR.1980311 (2004b:37032)

[2] Afraimovich, V. S., Bykov, V. V., Shilnikov, L. P., On attracting structurally unstable limit sets of Lorenz attractor type (Russian), Trudy Moskov. Mat. Obshch. 44 (1982), 150-212. MR656286(84a:58058)

[3] Bonatti, Ch., Viana, M., SRB measures for partially hyperbolic systems whose central direction is mostly contracting, Israel J. Math. 115 (2000), 157-193. MR.1749677 (2001j:37063a)

[4] Carballo, C., Morales, C., Pacifico, M. J., Homoclinic classes for generic $C^{1}$ vector fields, Ergodic Theory Dynam. Systems 23 (2003), no. 2, 403-415. MR1972228 (2004e:37031)

[5] Carballo, C., Morales, C., Pacifico, M. J., Maximal transitive sets with singularities for generic $C^{1}$ vector fields, Bol. Soc. Brasil. Mat. (N.S.) 31 (2000), no. 3, 287-303. MR 1817090 (2002b:37075)

[6] Gan, S., Wen, L., Nonsingular star flows satisfy Axiom A and the no-cycle condition, Invent. Math. 164 (2006), no. 2, 279-315. MR2218778 (2007j:37045)

[7] Gan, S., Wen, L., Heteroclinic cycles and homoclinic closures for generic diffeomorphisms, J. Dynam. Differential Equations 15 (2003), no. 2-3, 451-471. MR2046726 (2005b:37029)

[8] Gan, S., Li, M., Wen, L., Robustly transitive singular sets via approach of an extended linear Poincaré flow, Discrete Contin. Dyn. Syst. 13 (2005), no. 2, 239-269. MR2152388 (2006b:37056)

[9] Gan, S., Wen, L., Zhu, S., Indices of singularities of robustly transitive sets, Discrete Contin. Dyn. Syst. 21 (2008), no. 3, 945-957. MR2399444(2009a:37065)

[10] Guckenheimer, J., A strange, strange attractor, The Hopf bifurcation and its applications, Applied Mathematical Series 19, Springer-Verlag (1976). MR0494309 (58:13209)

[11] Guckenheimer, J., Williams, R., Structural stability of Lorenz attractors, Publ. Math. IHES 50 (1979), 59-72. MR556582 (82b:58055a)

[12] Hayashi, S., Diffeomorphisms in $\mathcal{F}^{1}(M)$ satisfy Axiom A, Ergodic Theory Dynam. Systems 12 (1992), no. 2, 233-253. MR.1176621 (94d:58081)

[13] Hayashi, S., Connecting invariant manifolds and the solution of the $C^{1}$-stability and $\Omega$-stability conjectures for flows, Ann. of Math. (2) 145 (1997), no. 1, 81-137. MR 1432037 (98b:58096)

[14] Hasselblatt, B., Katok, A., Introduction to the modern theory of dynamical systems. With a supplementary chapter by Katok and Leonardo Mendoza, Encyclopedia of Mathematics and its Applications, 54. Cambridge University Press, Cambridge (1995). MR1326374 (96c:58055)

[15] Hirsch, M., Pugh, C., Shub, M., Invariant manifolds, Lecture Notes in Mathematics, Vol. 583. Springer-Verlag, Berlin-New York, 1977. MR0501173 (58:18595)

[16] Liao, S., Qualitative theory of differentiable dynamical systems. Translated from the Chinese. With a preface by Min-de Cheng. Science Press, Beijing; distributed by American Mathematical Society, Providence, RI, 1996. MR1449640 (98g:58041)

[17] Mañé, R., A proof of the $C^{1}$ stability conjecture, Inst. Hautes Études Sci. Publ. Math. 66 (1988), 161-210. MR932138 (89e:58090)

[18] Mañé, R., An ergodic closing lemma, Ann. of Math. (2) 116 (1982), no. 3, 503-540. MR678479 (84f:58070)

[19] Mañé, R., Contributions to the stability conjecture, Topology 17 (1978), no. 4, 383-396. MR516217 (84b:58061)

[20] Metzger, R., Morales, C., Sectional-hyperbolic systems, Ergodic Theory Dynam. Systems 28 (2008), no. 5, 1587-1597. MR2449545(2010g:37045)

[21] Morales, C. A., Strong stable manifolds for sectional-hyperbolic sets, Discrete Contin. Dyn. Syst. 17 (2007), no. 3, 553-560. MR.2276427 (2008a:37036)

[22] Morales, C., Pacifico, M. J., A dichotomy for three-dimensional vector fields, Ergodic Theory Dynam. Systems 23 (2003), no. 5, 1575-1600. MR2018613(2005a:37030)

[23] Morales, C., Pacifico, M. J., Pujals, E. R., Singular-hyperbolic systems, Proc. Amer. Math. Soc. 127 (1999), no. 11, 3393-3401. MR1610761 (2000c:37034)

[24] Pliss, V. A., A hypothesis due to Smale, Differencial'nye Uravnenija 8 (1972), 268-282. MR0299909 (45:8957)

[25] Pugh, C., An improved closing lemma and a general density theorem, Amer. J. Math. 89 (1967), 1010-1021. MR0226670 (37:2257) 
[26] Shilnikov, L. P., Turaev, D. V., An example of a wild strange attractor, Sb. Math. 189 (1998), no. 1-2, 291-314. MR 1622321 (99d:58126)

[27] Shilnikov, L. P., Shilnikov, A. L., Turaev, D., Chua, L., Methods of qualitative theory in nonlinear dynamics. Part II, World Scientific Series on Nonlinear Science. Series A: Monographs and Treatises, 5. World Scientific Publishing Co., Inc., River Edge, NJ, 2001. MR.1884710 (2003d:37002)

[28] Wen, L., On the preperiodic set, Discrete Contin. Dynam. Systems 6 (2000), no. 1, 237-241. MR.1739926 (2001g:37035)

Instituto de Matématica, Universidade Federal do Rio de Janiero, P. O. Box 68530, 21945-970 Rio DE JANEIRO, BRAZIL

E-mail address: arbieto@im.ufrj.br

Instituto de Matématica, Universidade Federal do Rio de Janiero, P. O. Box 68530, 21945-970 Rio DE JANEIRO, BRAZIL

E-mail address: morales@impa.br 